\title{
TOPOLOgY AwARE LOAD BALANCING FOR GRIDS
}

\author{
Haitham Barkallah $^{1}$, Mariem Gzara ${ }^{2}$, Hanene Ben Abdallah ${ }^{3}$ \\ ${ }^{1}$ University of Sfax, MIRACL laboratory \\ ${ }^{2}$ Higher Institute of Computer Science and Mathematics of Mounastir, Tunisia \\ ${ }^{3}$ King Abdulaziz University, Jeddah, KSA
}

\begin{abstract}
Load balancing functionalities are crucial for best Grid performance and utilization. Accordingly,this paper presents a new meta-scheduling method called TunSys. It is inspired from the natural phenomenon of heat propagation and thermal equilibrium. TunSys is based on a Grid polyhedron model with a spherical like structure used to ensure load balancing through a local neighborhood propagation strategy. Furthermore, experimental results compared to FCFS, DGA and HGA show encouraging results in terms of system performance and scalability and in terms of load balancing efficiency.
\end{abstract}

\section{KEYWORDS}

Grid computing, meta-scheduling, distributed load balancing, topology-aware.

\section{INTRODUCTION}

Grid computing[1] extends existing distributed computing to a more unified and collaborative structure. It enables multiple computing resources, that are geographically distributed and owned by different individuals or organizations, to be logically coupled to form the illusion of a powerful super computer with infinite capacity[2].Scheduling and load balancingare essential grid software infrastructure services. Theseservices assigntasks to nodes(i.e. computing resources) and transfer tasks from overloaded to under-loaded nodesin orderto maximizeresource utilization on one hand,and minimizing the total task's execution time on the other hand. Considering the grid load balancing problem, several solutions have been proposed using different methods and strategies, e.g., [3][4].

Sofar, proposed methods can be classified into various categories depending on the point of interest: centralized $v s$. decentralized,decision taking [5 - 8], local $v s$. global optimization approach [9][10], static vs. dynamic strategies[11][3], adaptive $v s$. non-adaptive behavior[12][13], etc.

Among the most critical issues pertaining to grids is how to manage the resources[4]. In the proposed solution, the grid resources are organized according to a logical topology called theresource managementmodel[4]. This topology/model defines how the different grid entities communicate and work in order to achieve the received jobs/tasks. R. Buyyaet al. proclaimed that the choice of the right model for the resource management architecture plays a major role in its eventual (commercial) success [4].

The motivation behind this paperis based on the observation that the load balancing problem can be improved by simply improving the classical used models tree, star, and P2Pand further proposing a new one; a polyhedron sphere like model that was introduced for the first time in [14]. This logical model has the merit of providing optimized load balancing in a stateless context. It guarantees a high level of decentralization and scalability of the load balancing process leading tobetter resource utilization rates and minimum tasks execution time. Therefore,this work proposes adecentralizedtopology-awareload balancing solutionfor independent tasks in a grid environment 
named TunSys. The grid topology is constructed automaticallyin two steps. In the first step, a polyhedron topology, that has a sphere-like structure, is created. In the second step, the available resources are assigned to the nodes of the logical topology depending on the resource's physical characteristics. This structure is used to ensure a local neighborhood load balancing strategy. The presented method is dynamic, adaptive, cooperative, and non-preemptive.In addition, it uses a combination of two load thresholds: local and global.The experimental evaluation of the proposed model using the GridSim simulator has shown encouraging results in terms ofmakespan, system's scalability which can reach linear and supra-linear levels, and load balancing performanceoutperforming FCFS (First-come-first-served Algorithm)[5], DGA(Dynamic Genetic load balancing Algorithm) [7], and in most cases HGA (A hybrid load balancing strategy of sequential tasks for grid computing environments)[6].

The remainder of this paper is organized as follows: Section 2 gives a brief overview of the related work. Section 3 describes the proposed logical polyhedron topology. Section 4presents the proposed adaptive load balancing method. Section 5 discusses the simulation results. Finally, the paper's conclusion highlights some future research directions.

\subsection{RELATED WORK}

The Grid load balancing problem have been widely studied. Indeed, the literature contains several solutions using different strategies to solve the problem from different points of view.They can be classified according to different criteria: centralized $v$ s.decentralized, localvs.global, staticvs.dynamic, adaptivevs.non-adaptive, threshold-based $v$ s.threshold-independent, etc.Further details of the largely adopted taxonomy is well explained by Yagoubietal. In [15], an excellent survey on Grid resource allocation mechanisms is presented in [24, 25]. As examined in these detailed works, some of the proposed methods use agents [5], [11], [14], [26 - 29] to take profit from their decentralized, autonomous, and interacting character and thus avoid the "single point of failure". Some methods use genetic algorithms [5 - 7], [30] as a means to approximate solutions in polynomial complexity of the problem that can "evolve" during the operation of the parallel computing system.

Actually, this work focuses on two major characteristics: (i) the centralization vs. decentralization of the scheduling and load balancing processes, and (ii) their awareness of the network topology.

\subsubsection{Centralized vs. decentralized load balancing methods}

Many authors tried to find out whether it is better to decentralize or to centralize the load balancing process to fit the decentralized nature of the grid resources or to have a strong control on the tasks allocation decision.

In [6], Yajun Li and al. proposed a centralized load balancing scheme for sequential tasks. In [7] many issues are considered such as threshold policies, information exchange criteria, and interprocessor communication for a centralized solution. Li gang and al. [23] proposed two load balancing techniques according to the QoS requirement. The mean arrival rate is utilized by the central node to calculate the mean number of jobs in the waiting queues.

Liang Bai and al. [8] proposed to organize the grid nodes into multiple ant colonies. At the beginning, ants are distributed on computing nodes randomly. The ants move through computing nodes and assign the tasks based on pheromone and heuristic information until all tasks have been allocated. The load balancing approach is decentralized and collaborative. Another ant-like selforganizing mechanism is applied using agents by Junwei Cao [18], to perform complementary load balancing for batch jobs with no explicit execution deadlines. However, the main problem of these 
two solutions is the high number of exchanged messages implying high network resource consumption by the load balancing process.

Junwei Cao and al. work [5]used a combination of both intelligent agents and multi-agent approaches.Agents are organized into a tree: the broker heads the whole hierarchy and the coordinators head a sub-hierarchy. Each agent is a representative of a local grid resource and utilizes predictive application performance data and iterative heuristic algorithms to ensure local load balancing across multiple hosts.

\subsubsection{Topology aware vs. unaware load balancing methods}

The grid resources are physically interconnected to each other using network devices and cables forming a physical topology. While the system and workload model designate how the different parts of the system are logically organized and what kind of tasks it is supposed to achieve [4]. These models are key factors of the grid load balancing problem

In fact, the Grid must scale from few nodes up to a huge number of nodes. In addition, the system has to deal with the problems of node failure or unavailability and unexpected peaks of load, and it must be able to take all necessary corrective decisions. Furthermore, the part of resources consumed by the Grid load management process must be kept to an absolute minimum so as to maximize the resource utilization efficiency. Finally, the computing power consumed by a scheduler may be considered as a criterion of the scheduler selection [10]. The high amounts of computing and storage resources used by the decision making process would be considered as serious drawback like in [6].

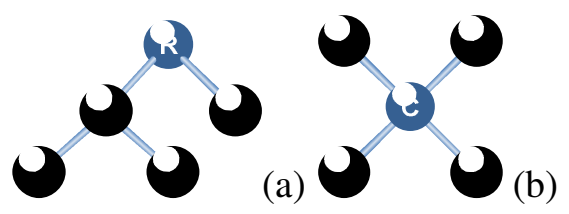

(c)

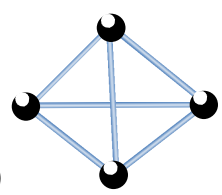

Figure .1 Tree (a), Star (b), and P2Psystem and workload model

The word Grid topology can describe the physical and/or logical arrangement of the nodes. It is often used to mean the logical topology, especially for open Grids where the Grid developers cannot predict the physical topology [2-4].

Many topology-aware scheduling and load balancing algorithms have been proposed which use different Grid topologies. They implement simple models like Tree [14 - 17], Peer-to-Peer [18 20], mesh [30], star [22, 23], etc. Even though these models are the most popular in the literature, real grid model scan integrate different sub-topologies at different levels.

Three cases are possible while constructing the Grid logical topology: It can be totally independent, partially dependent, or identical to the physical one. Camille Coti et al. [33]discussed how to organize communication patterns within applications with respect to the underlying physical topology. Because the various communication patterns induce different performances, the authors assume that an application ends up communicating back and forth between clusters if the inappropriate physical topology is used; such communication significantly impacts the performances. And in [34], the authors suggested a service for the delivery of dynamic 
performance information in Grid environments. They suggested that reflecting the real network topology in the application might improve the interface data structure.

This workproposes a new decentralized solution based on an innovative system model (referred to using the word "topology") in order to put in evidence the importance of the network. In fact, "the network is the computer" [35].The proposedtopology is decentralized, scalable, and well adapted to the grid load balancing process like in tree topology but doesn't "need the assistance of its direct parent" [24]. The nodes are interconnected to each other to form a sphere-like polyhedron constructed in two steps. Unlike the star topology, it does not need a central point to coordinate the load balancing process. It is "resilient to network traffic to deliver optimal performance" keeping the amount of network and computing resources needed for grid management in an absolute minimum since "the computing power consumed by a scheduler may be considered as a criterion of the scheduler selection"[36]. The next section presents the proposed topology: the structure construction, the resources assignment heuristic, and the local search algorithm used for topology optimization.

\subsection{The Polyhodron Grid Topology}

The Grid topology refers to the layout of connected nodes. It is the highest level defining how the nodes are physically and logically interconnected. The physical topology means the physical design of the Grid including the devices, location and network cable installation. The logical topology, on the other hand, refers to the way the different nodes and clusters constituting the Grid cooperate to execute the tasks.

The proposed polyhedron topology was inspired from the natural phenomenon of heat propagation in nature. In fact, heat spreads from a hot body to a cold body. This is called heat exchange process (or thermal equilibrium). The objects with sphere shape are a well-adapted form to heat spreading [37][38]. Heat spreads from the point of contact in a balanced manner until the total coverage of the spherical surface or total absorption. Similarly, during the proposed load balancing process, tasks are balanced from the overloaded nodes to the under loaded ones until equilibrium or task execution.

The proposed polyhedron topology is constructed in two steps: the polyhedron structure is created, then the grid nodes are assigned to the logical positions.

\subsection{Creation of the Grid polyhedron topology structure}

The proposed topology of the Grid has a polyhedron structure with a sphere-like form (0a)[39]. This structure facilitates the adoption of a local load balancing strategy.

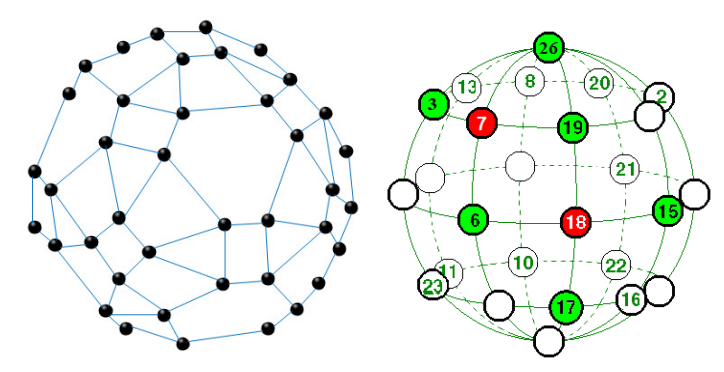

Figure .2 Grid Topology (a) Grid nodes organized as a polyhedron (b) Example of load situation 
The construction of this structure is performed in two steps: First, connectingthe nodes to form a flat 2D Grid like structure, then adding connections to obtain the final spherical like form. The flat $2 \mathrm{D}$ grid structure must be the nearest possible to a squared one.

To clarify this Gridtopology, let us start by describing the first "flat view" of the Grid. A 2D Grid composed of $p$ lines and $m$ columns where $\mathrm{m}=\left\lfloor\frac{n}{\lfloor\sqrt{n}\rfloor}\right\rfloor$ and $\mathrm{p}=\lfloor\sqrt{n}\rfloor$ with $\mathrm{n}=|\mathrm{X}|$ is constructed. The values of $m$ and $p$ are fixed so that the $\mathrm{m} \times \mathrm{p}$ grid is the nearest possible to a squared one. If $\mathrm{m}=\mathrm{p}$ then $n$ is a square and a squared grid is obtained. If $n=m \times p$ and $m \neq p$ then a grid of size $p \times m i s$ obtained. Otherwise, a partial $(p+1)^{\text {th }}$ line that contains only $k=n-(m \times p)$ nodes is added to the grid of $\mathrm{p}$ lines and $\mathrm{m}$ columns. 0 shows an example of the resulting 2D Grid for $n=10(0 \mathrm{a}), n=25(\mathrm{Ob})$. After node's connection, the polyhedron form of the Grid will have a spherical-like structure.
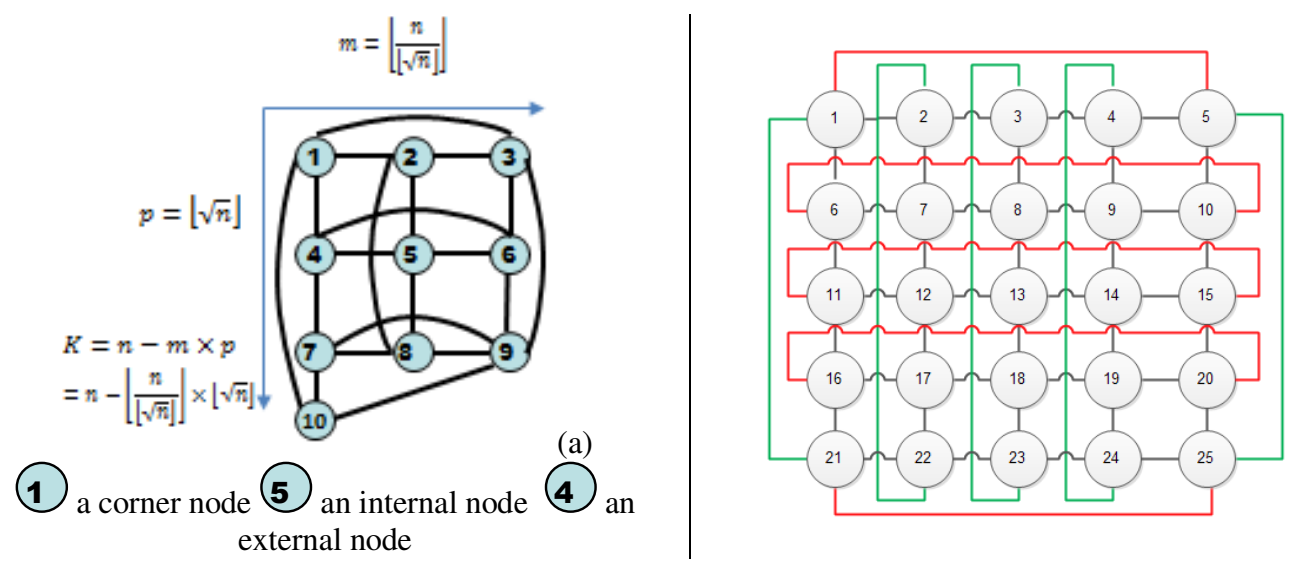

(b)

Figure .3 Example of the 2D view of the Grid: (a) $n=10$ (b)n=25

Afterward, each internal node is connectedto its four neighbors (instructions [4-7]). As a result, external nodes are connected to only three neighbors, whereas the Grid corners are connected only to two neighbors. Then external nodes at the top (respectively, at the right) areconnectedto nodes at the bottom (respectively, at the left) of the Grid (instructions [8 - 10]). The corner nodes at the extreme right of the two last lines are connected. Connections between the Grid nodes (graphically represented in $0[39]$ ) are resumed as follows:

- Instructions [4, 7]: Each node at position $(i, j)$ in the flat view where $1<i<p$ and $1<j<m$ is connected to the nodes at positions $(i-1, j),(i+1, j),(i, j-1)$ and $(i, j+1)$.

- If $n=m \times p$ then:

- each node at position $(1, \mathrm{j})$ is connected to the node $(\mathrm{p}, \mathrm{j})$

- each node at position $(\mathrm{i}, 1)$ is connected to the node $(\mathrm{i}, \mathrm{m})$

- If $n>m \times p$ then (instructions $[5,6,9,10]$ ):

- each node $(1, \mathrm{j})$ where $1 \leq \mathrm{j} \leq \mathrm{k}$ is connected to the node $(\mathrm{p}+1, \mathrm{j})$.

- $\quad$ each node $(1, \mathrm{j})$ where $\mathrm{k}<\mathrm{j} \leq \mathrm{m}$ is connected to the node $(\mathrm{p}, \mathrm{j})$.

- the node $(\mathrm{p}+1, \mathrm{k})$ is connected to the node $(\mathrm{p}, \mathrm{m})$ 
- the node $(p+1, k)$ is connected to the node $(p+1,1)$

- each node $(i, 1)$ where $1 \leq i \leq p$ is connected to the node $(i, m)$.

The construction of the Grid structure is handled in a time $O(n)$ for a Grid with $n$ nodes. In the worst case, the Grid has a total number of connections equal to:

$$
|R|=\left\{\begin{array}{l}
2 \times p \times m \quad \text { If }(n=p \times m) \\
n+m \times p+1 \quad \text { otherwise }
\end{array}\right.
$$

The algorithm complexity is equal to: $\Theta(n)=2 \times n+4$

\subsubsection{Resource assignment to the polyhedron topology}

It is beneficial to construct a logical topology that takes into account the characteristics of the physical one [34]. For example, when the network data transmission speed is high between two nodes, tasks are going to take less time to move from node to another. Thus the load balancing process will be improved if these nodes are neighbors (interconnected) in the logical topology.

The assignment of the resources to the nodes of the polyhedron logical topology depends on the physical network characteristics. In this context, the proposed topology reflects partially the physical one since its construction is based on an abstraction view of the physical network. To maximize the efficiency of the logical topology, resources having a high connection quality must be neighbors in the polyhedron topology.

To do so, a weight $G_{1 \mathrm{k}}$ that measures the connection quality between the resource $\mathrm{r}_{1}$ and the resource $r_{k}$ is assigned to each pair of resources $\left(r_{1}, r_{k}\right)$. The weight $G \partial_{r k}$ reflects different network parameters: baud rate, maximum transmission unit (MTU), and propagation delay. In [3] and [11], since the proposed solution is centralized, the authors quantified the connection quality of each node by dividing its transmission rate by the network bandwidth of the dispatcher. In [40] the network performance between two sites is calculated using two parameters: transmission delay representing the start-up cost and contention delays at intermediate links and a data transmission rate representing the available bandwidth.

The assignment of the nodes to the constructed logical topology depends on the weight between resources: if two resources have a high weight, then they are more susceptible to be assigned to connected nodes according to the logical topology and vice versa.

In the logical topology, each node $x_{i}$ is logically interconnected to $V\left(x_{i}\right)$ nodes then the weight between the resources assigned to the node $x_{i}$ and each node in $V\left(x_{i}\right)$ has to be maximized. For a given node $\mathrm{x}_{\mathrm{i}} \in \mathrm{X}$ the quantity $z_{i}$,which is the profit the node $\mathrm{x}_{\mathrm{i}}$ brings to the global grid topology, is computed as follows:

$$
Z_{i}=\prod_{x_{j} \in V\left(x_{i}\right)} \omega_{\pi_{i} \pi_{j}}
$$

Where $\mathrm{GJ}_{\pi \mathrm{i}, \pi \mathrm{j}}$ is the weight between the resource $\Pi_{\mathrm{i}}$ assigned to the node $\mathrm{x}_{\mathrm{i}}$ and the resource $\Pi_{\mathrm{j}}$ assigned to its neighbor $\mathrm{x}_{\mathrm{j}}$. A higher $\mathrm{z}_{\mathrm{i}}$ means a better network exchanging quality in the neighborhood of the node $x_{i}$. In $0 b$, the node $x_{18}$ neighbor's set is: $V\left(x_{18}\right)=\left\{x_{15}, x_{17}, x_{6}, x_{19}\right\}$. The profit the node $x_{18}$ brings to the whole topology is $Z_{18}=G_{\pi 18, \pi 15} \times \mathrm{GO}_{\pi 18, \pi 17} \times \mathrm{G}_{\pi 18, \pi 6} \times \mathrm{G}_{\pi 18, \pi 19}$. If the nodes $\left(x_{18}, x_{15}, x_{17}, x_{6}, x_{19}\right)$ are assigned to the resources $\left(r_{1}, r_{5}, r_{14}, r_{20}, r_{3}\right)$ then $Z_{18}=$ $Z_{1,5} \times Z_{1,17} \times Z_{1,16} \times Z_{1,15}$. In the computation of the network exchanging quality at node $x_{i}$, the product of the weight rather than the sum, is used to reduce the compensation between high values and low values of $\mathrm{GD}_{\mathrm{i}}$. 
Furthermore, the assignment of the resources to the nodes of the logical topology must maximize the profit of the whole Grid which is equal to the sum of the profits of all the nodes. The assignment of the resources to the nodes of the logical topology aims at maximizing the objective function:

$$
Z=\sum_{k=1}^{n} Z_{k}
$$

Asolution to this problem is a permutation $\Pi=\left(\Pi_{1}, \Pi_{2}, \ldots, \Pi_{n}\right)$ where $\Pi_{i}$ is the resource assigned to the logical node $\mathrm{x}_{\mathrm{i}}$. So the number of possible solutions is equal to $\mathrm{n}$ !.

Note that the resource assignment to the logical nodes of the polyhedron is a combinatorial optimization problem which can be resolved either by an exact method like simplex algorithm, dynamic programming, or by approximate methods. In this case, the goal is a solution that maximizes as best as possible the objective function $\mathrm{Z}$ in a polynomial time. The studied problem needs to be solved (only have a good quality solution) in real (short) time and using the absolute minimum of resources. Consequently, it was considered useful to solve it heuristically by the use of a constructive algorithm followed by a local search to improve the obtained solution. In this case, the optimality of the solution cannot be guaranteed. However, the time required to obtain this solution will be much lower and can even be dead-lined (obviously in this case the quality of the solution strongly depends on the time allowed for the algorithm to get it).

\subsubsection{The construction heuristic}

The construction heuristic starts from an empty solution $\pi=\emptyset$. It selects randomly a resource $r$ and a node $x$ and assigns $r$ to $x$. Then, iteratively, it selects one occupied node $x$ of the logical topology still having idle neighbors according to the logical topology and assigns to them the most profitable resources according to network quality criterion $Z_{x}$. The algorithm ends when all the resources are assigned thus all the logical nodes are occupied.

Let $\mathrm{R}=\left\{\mathrm{r}_{1}, \ldots ., \mathrm{r}_{\mathrm{n}}\right\}$ and $\mathrm{X}=\left\{\mathrm{x}_{1}, \ldots, \mathrm{X}_{\mathrm{n}}\right\}$ denote respectively the set of resources and nodes. Initially, the set of idle nodes $I X=X$ and the set of free resources $F R=R$. A node $\mathrm{x}$ of the logical topology is idle if neither resource is assigned to it. A resource $r$ is free if it is affected to any logical node. A resource $r$ is assigned to exactly one logical node and a logical node receives a sole resource.

The algorithm starts by selecting a random resource $\mathrm{r}_{\mathrm{i}}$ from $F R(\mathrm{i} \in<1, \mathrm{n}>)$ and a random node $\mathrm{x}_{\mathrm{j}}$ $(\mathrm{j} \in<1, \mathrm{n}>)$. A partiel solution $\pi=\left\{\pi_{i j}\right\}$ is obtained and the set of idle nodes and free resources are updated $\left(F R=F R \backslash\left\{r_{i}\right\}\right)$ and $\left(I X=X \backslash\left\{x_{j}\right\}\right)$. Given a partial solution $\pi$, a given node $x$ have three possible states:

- The node $x$ is idle i.e. no resource is affected to the node $x$.

- The node $\mathrm{x}$ is occupied by a resource but at least one of its local neighbors is idle. The node $\mathrm{x}$ is, then, still unlocked.

- The node $\mathrm{x}$ is locked i.e. the node $\mathrm{x}$ and all its neighbors are occupied

The set of unlocked nodes is stored in the list Q. The algorithm affects iteratively resources to unlocked nodes until all nodes are locked. The most profitable unlocked node is selected and the free resources are assigned to its idle neighbors. 


\section{Algorithm 1: The construction heuristic}

1) $\quad F R:=\left\{x_{j} ; j \in<1, n>\right\}$

2) IX : $:=\left\{x_{j} ; j \in<1, n>\right\}$

3) $\quad r:=\operatorname{random}(\mathrm{FR})$

4) $x:=$ random (IX)

5) $\quad \Pi_{\mathrm{i}}:=\varphi$

6) $\quad \Pi_{\mathrm{i}}:=\Pi \cup\left\{\Pi_{\mathrm{rx}}\right\}$

7) $Q_{\mathrm{i}}:=\{\mathrm{x}\}$

8) $\quad \mathrm{FR}:=\mathrm{FR} \backslash\{\mathrm{x}\}$

9) IX $:=\operatorname{IX\backslash } \backslash\{\mathrm{r}\}$

10) While $(\mathrm{Q} !=\varphi)$ Do

$\mathrm{x}_{\mathrm{j}}:=\max \mathrm{Z}_{\mathrm{k}}, \mathrm{k} \in \mathrm{Q}$

update FR

update IX

update Q

update $\Pi$

EndWhile.

The complexity of the construction heuristic is $\Theta\left(n^{2}\right)$.

\subsubsection{Local search}

After obtaining an initial solution $\Pi_{0}$ with the profit $Z_{0}$, it is improved through local search. During each local search iteration, the algorithm local search tries to find all possible permutations between the resources assigned to two distinct nodes $x_{i}$ and $x_{j}$ that can lead to a new solution $\Pi_{\text {new }}$ such that $Z_{\text {new }}>Z_{\text {old }}$. The best generated solution is kept as initial solution at the next iteration. The grid system keeps trying to find good permutations till impossibility of improving the achieved solution. It is possible that the finally obtained solution is not the optimal one. Indeed, the algorithm looks for local minima. It converges rapidly. This keeps the used algorithm in harmony with one of the proposed topology's goal which is to minimize the grid's system load and give resources privilege to the user's jobs. The complexity of each iteration of the local search is $O\left(n^{2}\right)$ since the size of the neighborhood is $C_{n}^{2}$ and the cost of the fitness variation is:

$$
O(2 *|V(x)|)
$$

A solution of the problem is coded as an assignment vector of size $\mathrm{n}$ ( $\mathrm{n}$ is the number of resources). $\Pi[i]=j$ means that the resource $r_{j}$ is assigned to node $x_{i}$. The neighborhood of the solution $N(\Pi)$ is composed of all assignment vectors obtained after the permutation of two members $\Pi i i]$ and $\Pi j]$ $(i \neq j) .\left(|N(\Pi)|=C_{n}^{2}\right)$.

For each generated solution $\Pi$, the variation of the cost is computed.

$$
\begin{gathered}
\Delta_{z}=Z(\Pi)-Z\left(\Pi_{0}\right)= \\
\left(Z_{i}^{\Pi}+Z_{j}^{I}\right)-\left(Z_{i}^{\Pi_{0}}+Z_{j}^{\Pi_{0}}\right)+\sum_{k \in V(i)}\left(Z_{k}^{\Pi_{0}}-Z_{k}^{\Pi_{0}} * \frac{\omega_{k j}}{\omega_{k i}}\right)+\sum_{k \in V(j)}\left(Z_{k}^{\Pi_{0}}-Z_{k}^{\Pi_{0}} * \frac{\omega_{k i}}{\omega_{k j}}\right)
\end{gathered}
$$

$Z_{i}^{\Pi}$ is the local network quality of node $i$ in solution $\Pi$ if $\Delta_{z}>0$. The local search algorithm is given in the following algorithm. As a result, the cost of the generation and the evaluation of the neighborhood is $O\left(n^{2}\right)$. 


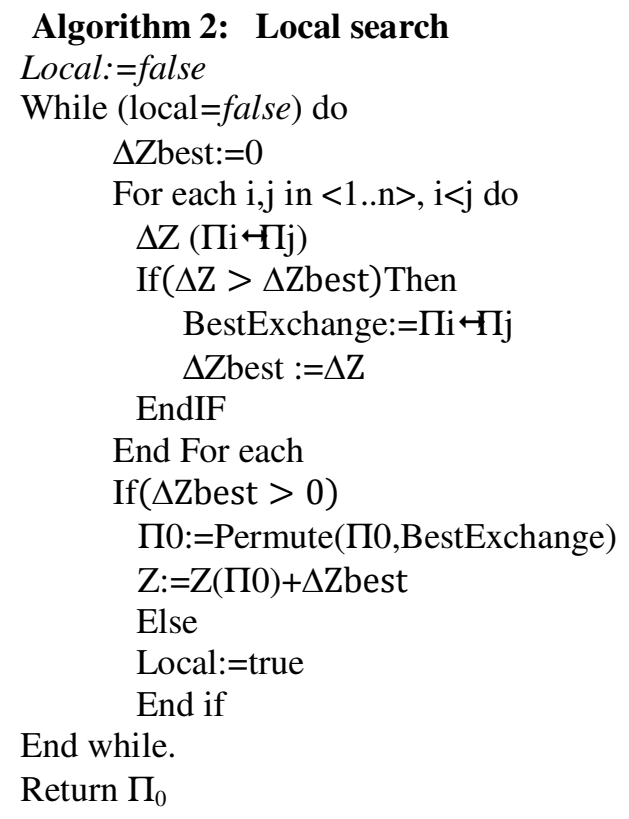

\section{ADAPTIVE LOAD BALANCING}

TunSys uses an adaptive andfully decentralized load balancing strategy. The workload is evaluated periodically both globally at the grid level and locally at the node's level and the load thresholds are adapted. Due to polyhedron logical topology, every node has a set of neighboring nodes. So, the overload propagates from the overloaded nodes to their neighbors and from neighbors to neighbors until a balance of the workload is reached. When the overall grid workload is high, the local and global thresholds are updated so as the nodes can absorb more jobs and the workload balance can be realized overall the grid.

Each node makes local decisions according to its state and the state of its neighbors. In case of unexpected peak of load, the node initiates a sender side load balancing process and the jobs are routed to relatively idle nodes in its local neighborhood. The scheduling process is also based on local decision and is fully decentralized. Each node creates schedules that consider local objectives and constraints within the boundaries of the overall system objectives.

\subsection{Global and local load thresholds}

Let $\mathrm{Q}_{\mathrm{i}}, \mathrm{i} \in<1, \mathrm{n}>$ be the waiting queue of node $\mathrm{I}_{\mathrm{i}}$ and $\left|\mathrm{Q}_{\mathrm{i}}\right|$ be the length of the tasks waiting to be executed on the node $I_{i}$. Let $V(i)$ be the set of neighbors of the node $I_{i}$ according to the logical topology.

The node's load is evaluated using a load upper bound $\alpha_{\mathrm{i}}$ If the node's queue $\left|\mathrm{Q}_{\mathrm{i}}\right|$ length exceeds $\alpha_{\mathrm{i}}$ it is considered as an overloaded node $\left(\left|\mathrm{Q}_{\mathrm{i}}\right|>\alpha_{\mathrm{i}}\right)$. Initially, for a given node $\mathrm{x}_{\mathrm{i}}$, the upper bound is evaluated according to the global Grid load thresholds $\alpha$ and $\beta$ and the number of its processing elements $\left|\mathrm{PEs} \mathrm{s}_{\mathrm{i}}\right|$ :

$$
\alpha_{\mathrm{i}}=\alpha \times\left|\mathrm{PES}_{\mathrm{i}}\right|
$$

The thresholds arepresents the upper bound of the global Grid load. It represents the load indicator of the waiting queues at the Grid level. Initially, $\alpha=2$; later the system periodically collects 
information about the current load and waiting queue length of all the Grid nodes, and it adapts $\alpha$ dynamically according to the resource load variation as follows:

$$
\left.\alpha_{\text {new }}=\alpha_{\text {old }} *(1+\gamma) \quad \text { while }(1+\gamma) \in\right] 0,2[\text { and } \alpha>1
$$

Where $\gamma$ is an indicator of the current workload state and is computed according to the following formula:

Where:

$$
\gamma=\frac{\left|\left\{x_{i} / \frac{\left|Q_{i}\right|}{P E S_{i}}>M+E\right\}\right|}{|X|}-\frac{\left|\left\{x_{i} / \frac{\left|Q_{i}\right|}{P E S_{i}} \leq M-E\right\}\right|}{|X|}
$$

- $\mathrm{M}$ is the mean of grid node's load $\mathrm{M}=\mathrm{AVG}\left(\left|\mathrm{Q}_{\mathrm{i}}\right|, \mathrm{x}_{\mathrm{i}} \in \mathrm{X}\right)$

- $\mathrm{E}$ is the standard deviation of the grid node's load $\mathrm{E}=\mathrm{STD}\left(\left|\mathrm{Q}_{\mathrm{i}}\right|, \mathrm{x}_{\mathrm{i}} \in \mathrm{X}\right)$

A given node is considered as overloaded if the length of its queue per processing element is more than a standard deviation over the load's mean. A given node is considered as under-loaded if the length of its queue per processing element is less than the loads mean minis the standard deviation of the node's load.

The value of $\gamma$ is in the intervall ]-1, 1[. A positive value shows that the majority of the nodes are overloaded $(\gamma>0)$ where a negative value shows that there are more under-loaded nodes than overloaded ones $(\gamma<0)$. The combination of the two measures (average and standard deviation) makes the threshold $\alpha$ sensible not only to the grid's peak of load (when the grid load average increases) but also to the spread out of this load.

When the value of $\gamma$ is positive, local peak of loads are detected and the value of the global threshold is increased and thus the local ones. So, the under-loaded nodes can accept the reception of more jobs and the most overloaded nodes will be discharged in priority.

When the value of $\gamma$ is negative, the value of the global threshold is decreased and intuitively the local one's. The state of some node's may change from the underload to the overload state and the load propagation process may be inhibited. In fact, if the proportion of under-loaded nodes is high, the load have to be balanced overall the nodes so as to speed up the response time overall the grid.

\subsubsection{Load balancing process}

The loadbalancing process is triggered or stopped in any node $x$ of the Grid depending on the following scenarios:

- A new task arrives to $\mathrm{x}_{\mathrm{i}}$ : When $\mathrm{x}_{\mathrm{i}}$ receives a task, its local workload queue is reevaluated. If $x_{i}$ is already overloaded or if the acceptance of this task will make it overloaded $\left(\left|\mathrm{Q}_{\mathrm{i}}\right|>\alpha_{\mathrm{i}}\right)$, then $x_{i}$ triggers the load balancing process; otherwise the task is inserted into the waiting queue of $x_{i}$.

- $\mathrm{x}_{\mathrm{i}}$ receives a new value of the global threshold $\alpha$ : $\mathrm{x}_{\mathrm{i}}$ updates its local threshold $\alpha_{\mathrm{i}}$ andchecks its new load state:

- If $\alpha$ decreased and if $x_{i}$ becomes over-loaded $\left(\left|\mathrm{Q}_{\mathrm{i}}\right|>\alpha_{\mathrm{i}}\right)$, then $\mathrm{x}_{\mathrm{i}}$ triggers the load balancing process in the sender initiated mode.

- If $\alpha$ increased, then the waiting queue $\mid \mathrm{Q}_{\mathrm{i}} \mathrm{I}$ can accept more tasks. Therefore, if the load sender had initiated the balancing process, then it is stopped. 
Generally, each load balancing and load sharing algorithm can be defined by three rules: the location rule, the distribution rule and the selection rule [41] :

- The location rule defines which grid resources will be included in the balancing process.

- The distribution rule decides how to redistribute the workload among available nodes.

- The selection rule selects tasks in an overloaded node for reassignment to an under-loaded node.

In the proposedsolution, the logical neighborhood relation defined by the polyhedron topology is used to perform the load balancing. This topology has a spherical like structure and a given node $\mathrm{x}$ of the grid has $\mathrm{V}(\mathrm{x})$ neighbors. The overload is transferred from neighbors to neighbors until complete absorption. The load balancing process is decentralized.

Each node xi ( $i \in<1, n>$ ) checks its load state based on its current waiting queue and local threshold $\alpha i$. Periodically, each node sends information about its load level to its neighbors. In fact, a given node maintains neighborhood's Load table (NL). Such table represents a snapshot of a small part of the grid. Each node perceives its load state and those of its neighbors and decides on the lunching of the balancing process based on this local vision of the load spread. This local decision process applies the following rules:

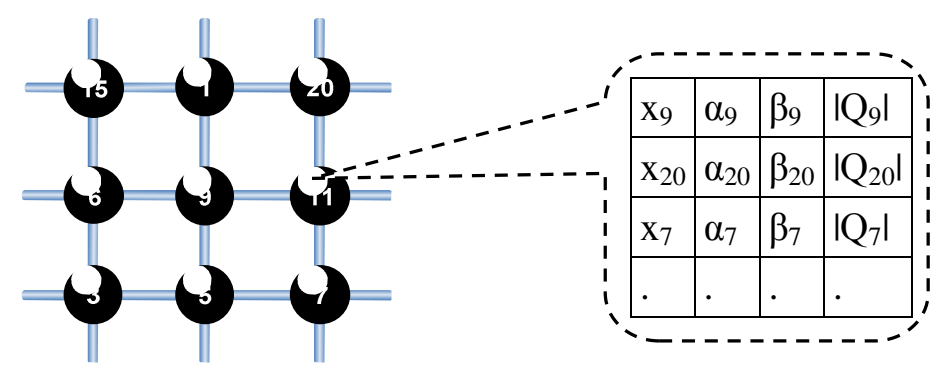

Figure.4 NL table for node $\mathrm{x}_{11}$.

Location rule: In case a node $x_{i}$ becomes overloaded, it tries to find an under-loaded node $x_{j}$ from its neighbors $\mathrm{V}\left(\mathrm{x}_{\mathrm{i}}\right)$ ready to receive some jobs. In the example of $0 \mathrm{~b}$, node 18 for instance, can send jobs only to nodes $19,17,6$ and 15 .

Distribution rule: The chosen neighbor is the least loaded one. If all elements of $\mathrm{V}\left(\mathrm{x}_{\mathrm{i}}\right)$ are overloaded ( $x_{i}$ and all its neighbors), then $x_{i}$ does not wait until one of its neighbors becomes under-loaded. It directly sends it to the least loaded one which in turn has to balance it to one of its neighbors.

Selection rule: In this work, an Early and Late [11] technique is applied for the queue management in order to adapt task execution to non-deterministic nature of the Grid computations. At the node level, tasks in its waiting queue $\left|\mathrm{Q}_{\mathrm{i}}\right|$ are sorted according to their submission time; the oldest tasks are favored for more fairness. In case a task had been delayed for being balanced from node to node, it is fostered while waiting in the queue. In case a task has to be transferred to an under-loaded node, the last one in the queue is selected since it is the last to be submitted to the Grid.

Accordingly, the node's local neighborhood and the equilibration of overload are relayed from neighbors to neighbors-imitating the heat propagation principle. The situation of starvation can 
be avoided whenever there is the overload spread from overloaded part of the grid to the underloaded one.

\section{EXPERIMENTATION}

The GridSim simulator [42]was used and extended with several classes and interfaces to fulfill specific requirements of TunSys and to take into account the semantics of node description and different resource types. The proposed solution have been simulated in the context of a private Grid context as defined in [17]. The evaluation focus on the workload balance, the completion time and the scalability. Each experiment had been repeated 10 times. The results are discussed in terms of the mean values.

\subsection{The evaluation metrics}

The mean square deviation criteria is used to evaluate the resource load balancing performance. It is computed as follows:

$$
\sigma=\sqrt{\frac{\sum_{\mathrm{i}=1}^{\mathrm{m}}\left(\mathrm{P}_{i}-\overline{\mathrm{P}}\right)^{2}}{\mathrm{~m}}}
$$

where $\mathrm{P}_{\mathrm{i}}, \overline{\mathrm{P}}$ denotes the individual node utilization and the average node utilization and $\mathrm{m}$ is the number of resources. This metric indicates how well the loads are balanced across all the nodes involved in a grid system. The lower the measurement of this performance metric is, the better the performance of load balancing is. When the value of the mean square deviation is high, a large gap between the node's workloads is deduced and thus picks of overloads and valleys of underloads may exist.

To verify if the load balancing process is optimizing the completion time, the makespanmetric have been used which represents the latest completion time among all the tasks, i.e. the derived maximum load among all the computing nodes:

$$
\max _{i \in[1, n]}\left(L_{i}+\sum_{i \in[1, q]} E T_{i j}\right)
$$

where $\mathrm{ET}_{\mathrm{ij}}$ is the execution time of task $T_{i}$ on node $C_{j}$ when $T_{i}$ is assigned to node $C_{j}$, otherwise $\mathrm{ET}_{\mathrm{ij}}$ is 0 , and $\mathrm{n}$ is the number of tasks.

\subsubsection{The simulation scenario}

First many papers have been analyzed in order to choose an experimentation scenario detailed enough to be reconstituted and to suit the limited hardware and software resources. The goal is also to compare this work to other proposed solutions from the literature. The proposed solution was compared to:

- First-come-first-served algorithm (FCFS) [5]

- Dynamic Genetic load balancing Algorithm (DGA) [7]

- A hybrid load balancing strategy of sequential tasks for grid computing environments (HGA) [6] 
The private Grid is composed of 5 nodes. The Grid resources are physically organized into a tree structure like in [26]. The simulation parameters (0) mentioned in [6] have been followed.

Table 1.Experimental parameters.

\begin{tabular}{|l|l|}
\hline Parameters & Values \\
\hline Number of computing nodes & 5 \\
\hline Capacity of nodes & 1 \\
\hline Number of tasks & 250 \\
\hline Mean Inter-arrival Time & $1 \mathrm{~s}$ \\
\hline Mean Computation length & $5 \mathrm{~s}$ \\
\hline
\end{tabular}

Several assumptions are made for the simulation:

- Tasks arrive and enter into the RMU, according to a Poisson process with rate $\lambda$.

- The expected computation lengths of tasks are assumed to follow an exponential distribution with a mean $\chi$.

- The average capability $\mathrm{C}_{\mathrm{i}}$ of node $\mathrm{i}$, which is the relative ratio of its capability.

- Let $\rho$ be the average system utilization factor for this simulation, which is the average task arrival rate divided by the average task processing rate [43]. According to this definition, the task computation length $\chi$ is adjustedin order to get the desired $\rho$.

- All the nodes used in the experiments, have the same processing capacity.

- The grid physical topology used in this experiment has a tree structure [26].

\subsubsection{Evaluation of the system robustness}

In this section, the system's performance is studied using the makespan metric and then the results are compared to the ones published in [6]. The makespan metric is widely used in the literature in order to measure the system robustness. In this experiment, the number of tasks is varied from 100 to 500 with an interval of 50. The mean computation length of the tasks $\chi$ is also varied to keep the average system utilization factor $\rho$ at a value of 1.4.

Table .2 Measurement lists of makespan metric

\begin{tabular}{|l|l|l|l|l|}
\hline \multirow{2}{*}{ Tasks } & \multicolumn{4}{|c|}{ Makespan } \\
\cline { 2 - 5 } & DGA & FCFS & HGA & TunSys \\
\hline 100 & 100,90 & 94,10 & 90,60 & $\mathbf{8 5 , 0 9}$ \\
\hline 150 & 137,60 & 132,80 & 129,20 & $\mathbf{1 2 4 , 0 4}$ \\
\hline 200 & 196,60 & 184,90 & $\mathbf{1 8 1 , 0 0}$ & 183,01 \\
\hline 250 & 230,10 & 230,40 & $\mathbf{2 2 2 , 5 0}$ & 235,00 \\
\hline 300 & 275,70 & 269,10 & 265,90 & $\mathbf{2 6 1 , 0 3}$ \\
\hline 350 & 326,70 & 315,40 & $\mathbf{3 1 2 , 0 0}$ & $\mathbf{3 1 2 , 1 3}$ \\
\hline 400 & 361,90 & 356,40 & 352,40 & $\mathbf{3 5 2 , 0 0}$ \\
\hline 450 & 422,10 & 413,10 & $\mathbf{4 0 7 , 3 0}$ & 414,09 \\
\hline 500 & 466,00 & 459,20 & $\mathbf{4 5 6 , 4 0}$ & $\mathbf{4 6 2 , 0 7}$ \\
\hline
\end{tabular}

As shown in 0, the makespan of tasks increases when the number of tasks grows since more tasks are seeking to be executed. The proposed method TunSys outperforms DGA and FCFS in all cases. In addition, it gives good results compared to HGA even though TunSys uses a simple Random scheduling policy and focuses on system load balancing while HGA integrates scheduling and load balancing optimization. According to these results, TunSys is able to accommodate and thus performs more robustly than DGA and FCFS and HGA in most cases. 


\subsubsection{Load-Balancing performance}

In order to test the load balancing performance of these algorithms, two scenarios are used: homogeneous nodes and heterogeneous nodes with $\lambda=1.0$, average Poisson distribution $=0.96$, total tasks $=100$ and the utilization factoris varied between 0.8 to 1.5 by varying the tasks' length.

\subsection{Performance under homogeneous nodes}

All the nodes have the same processing capacity. The mean computation length $\chi$ is varied between 4 seconds and 7.5 seconds, and the average system utilization factor from 0.8 to 1.5 . The tests are repeated ten times in order to get mean values of the load balancing.

The results ( 0 and Error! Reference source not found.and 0 ) show that, under homogeneous nodes scenario, the proposed method shows better stability than FCFS and HGA. In fact, even though the system utilization factor changes the load, distribution keeps stable (between 0.04 and 0.05 ) and varying in a limited interval. While for HGA, for example, the square deviation of node utilization varies between 0.01 and 0.126 .

Furthermore, when the utilization factor is less than 1.1, TunSys outperforms clearly the other two solutions. However, when it becomes greater than 1.1, it is exceeded by FCFS and HGA. This can be explained by the fact that TunSys uses local thresholds to classify the state of a node as overloaded or not. So, the load balancing process is triggered only for nod nodes having their waiting queue length higher than the workload thresholds.

Table .3 The square deviation of node utilization under homogeneous nodes scenario

\begin{tabular}{|c|l|l|l|}
\hline Average Utilization Factor & TunSys & FCFS & HGA \\
\hline $\mathbf{0 . 8}$ & $\mathbf{0 . 0 4 7}$ & 0.126 & 0.126 \\
\hline $\mathbf{0 . 9}$ & $\mathbf{0 . 0 5 0 2}$ & 0.072 & 0.072 \\
\hline $\mathbf{1 . 0}$ & $\mathbf{0 . 0 4 4}$ & 0.054 & 0.052 \\
\hline $\mathbf{1 . 1}$ & 0.049 & 0.046 & $\mathbf{0 . 0 4 2}$ \\
\hline $\mathbf{1 . 2}$ & 0.0458 & 0.038 & $\mathbf{0 . 0 3 6}$ \\
\hline $\mathbf{1 . 3}$ & 0.0498 & 0.03 & $\mathbf{0 . 0 2 8}$ \\
\hline $\mathbf{1 . 4}$ & 0.046 & 0.034 & $\mathbf{0 . 0 1 8}$ \\
\hline $\mathbf{1 . 5}$ & 0.047 & 0.034 & $\mathbf{0 . 0 1}$ \\
\hline & & & \\
\hline
\end{tabular}

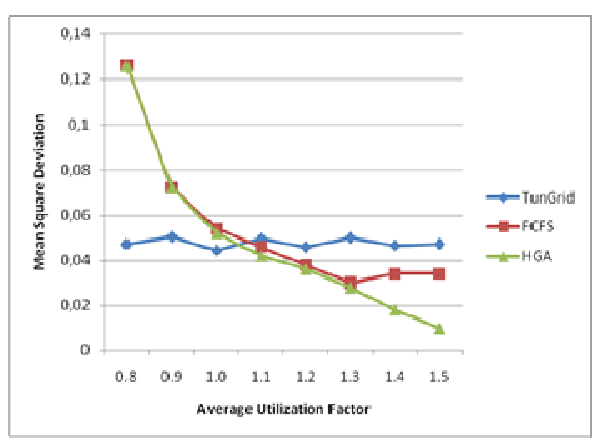

Figure 5 The square deviation of nodes' utilization under homogeneous nodes scenario

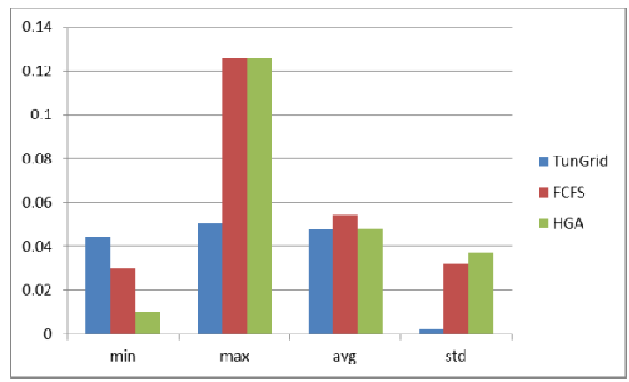

Figure 6. Minimum, Maximum, Average and Standard deviation of the square deviation of nodes' utilization under homogeneous nodes scenario 


\subsubsection{Performance under heterogeneous nodes}

In this case, the system performance is tested under a heterogeneous Grid system. Two nodes are randomly selected and fixed their relative capabilities which is doubled by the value of the others. The mean computation length $\chi$ is varied between 5.6and 10.5 seconds and the average system utilization factor from 0.8 to 1.5 . The simulations are repeated ten times in order to get mean values of the load balancing.

Once again, under heterogeneous nodes scenario (0), TunSys shows better performance and stability than FCFS and HGA. In fact, the load distribution varies in a limited interval between 0.074 and 0.091 even though the system utilization factor changes. It is only when it becomes greater than 1.3 that HGA outperformsTunSys. Again, this is due to the fact that the proposed solution focuses on the network in order to take the load balancing decisions not the utilization factor which makes it more efficient and stable to load variations.

Table .4 The square deviation of node utilization under homogeneous nodes scenario

\begin{tabular}{|c|l|l|l|}
\hline Average Utilization Factor & TunSys & FCFS & HGA \\
\hline $\mathbf{0 . 8}$ & $\mathbf{0 . 0 9 1}$ & 0.3208 & 0.2541 \\
\hline $\mathbf{0 . 9}$ & $\mathbf{0 . 0 8 1}$ & 0.3 & 0.1958 \\
\hline $\mathbf{1 . 0}$ & $\mathbf{0 . 0 8 6}$ & 0.2791 & 0.1416 \\
\hline $\mathbf{1 . 1}$ & $\mathbf{0 . 0 8 3}$ & 0.2708 & 0.1083 \\
\hline $\mathbf{1 . 2}$ & $\mathbf{0 . 0 8 8}$ & 0.2666 & 0.1041 \\
\hline $\mathbf{1 . 3}$ & 0.0747 & 0.2625 & $\mathbf{0 . 0 6 6 6}$ \\
\hline $\mathbf{1 . 4}$ & 0.087 & 0.2541 & $\mathbf{0 . 0 5 4 1}$ \\
\hline $\mathbf{1 . 5}$ & 0.0857 & 0.2539 & $\mathbf{0 . 0 3 7 5}$ \\
\hline
\end{tabular}

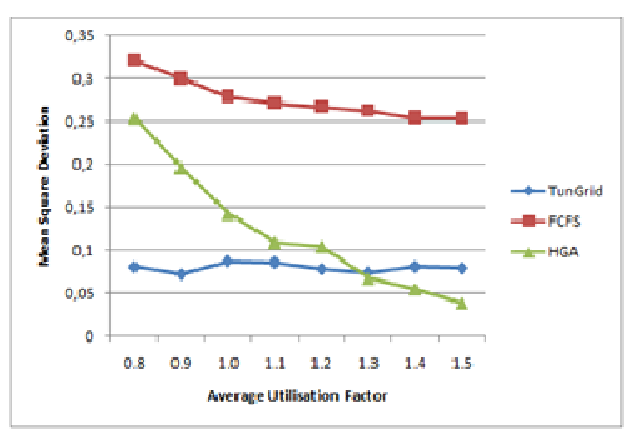

Figure .7 The square deviation of nodes' utilization under heterogeneous nodes scenario

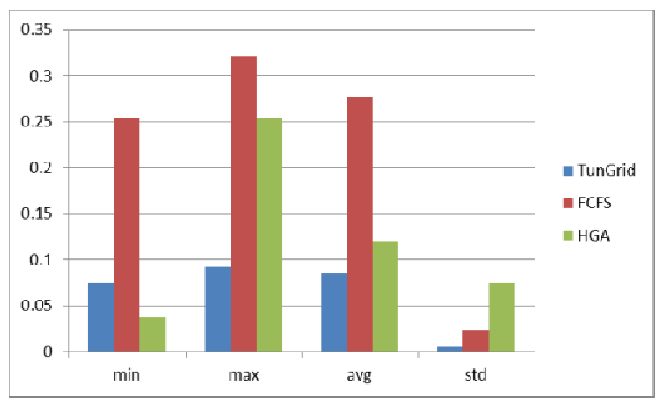

Figure8 Minimum, Maximum, Average and Standard deviation of the square deviation of nodes' utilization under heterogeneous nodes scenario 


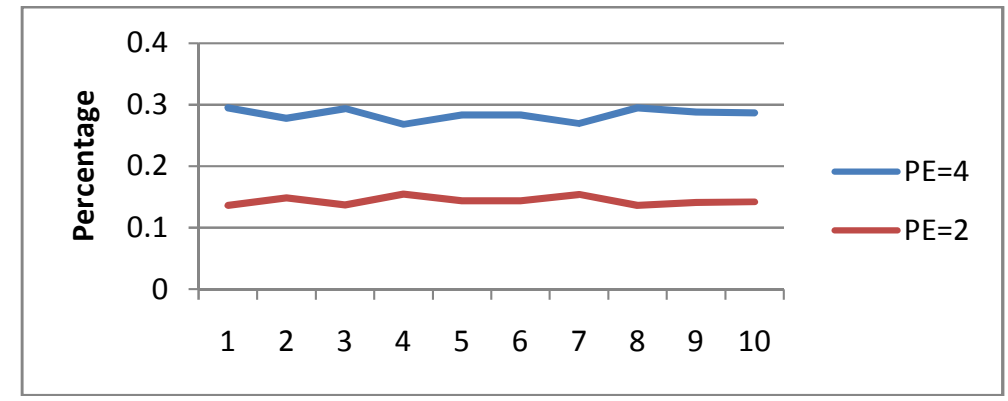

Figure 9. Average percentage of executed tasks per resource considering resources with $\mathrm{PE}=2$ vs. resources with $\mathrm{PE}=4$.

0 , presents the average percentage of executed tasks per resource. The goal is to study the load distribution in case some of the resources are more powerful than the rest. The results show that the load is well redistributed among the grid. The resources with $P E=4$ are executing more tasks (almost two times) than the resources equipped with $\mathrm{PE}=2$ even though TunSysuses a blind scheduling policy.

\subsubsection{Evaluation of the scalability}

The experimentation scenario focuses on the system's scalability. The system's utilization factoris fixed at 0.8 while the number of resources is varied as follows: 10 - 20 - 30 - 40 - and 50 and the number of simulated tasks $100-1000-10000$ with a mean computation length $\chi$ varying between 5.6 and 10.5 seconds. The remaining parameters are kept the same as in the first experimentation scenario.The resources are physically interconnected using the same topology as in [26]. However, they are arbitrary interconnected to the routers in order to reduce the effect of the physical topology.TunSysis simulated under two cases: homogeneous resources and heterogeneous resources. In the case of heterogeneous resources, $50 \%$ of the nodes are randomly selected and fixed their relative capabilities (number of processing elements) to twice the value of the others.Finally, each experiment is repeated 10 times and the discussed results are the mean values so that the effects of the random parameters on the resultscan be reduced.

Opresents the average system makespan while varying the number of tasks and resources. As expected, the makespan value decreases by $38 \%$ up to $84 \%$ in the case of homogeneous resources and by $43 \%$ up to $84 \%$ in the case of heterogeneous resources (0). As shown in 0 the makespan value decreases almost linearly by $34 \%$ in average every time the number of resources increases by 10 . Except in case of 100 tasks, the system does not scale well and the average speedup is about $11 \%$ which is absolutely normal for two reasons. Firstofall, the number of executed tasks is very limited, and second, the used blind scheduling policy which is affecting some of these tasks to distant resources neither consider the network transmission time nor forecast the load of the most close resources to the user submitting the tasks. As a conclusion, there is a linear and supra-linear scalability in case of 1000 and 10000 tasks. 
International Journal of Grid Computing \& Applications (IJGCA) Vol.8, No.1/2/3, September 2017

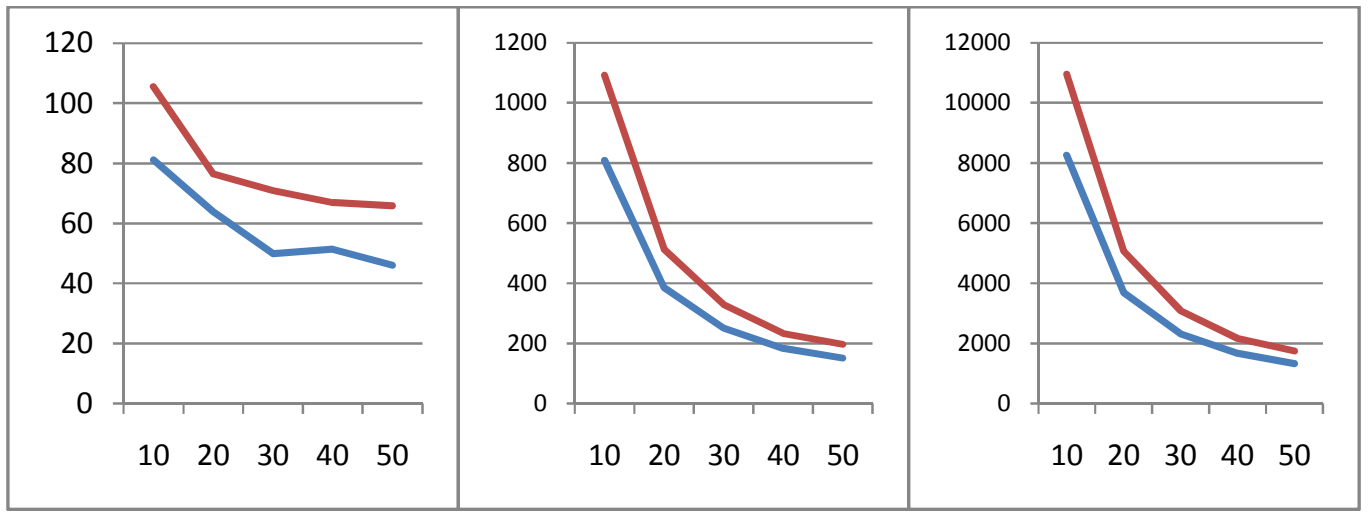

Figure 10. Average makespan using 100, 1000, and 10000 tasks, 10-50 resources, and homogenous ( ) vs. heterogeneous ( $\quad$ ) resources.

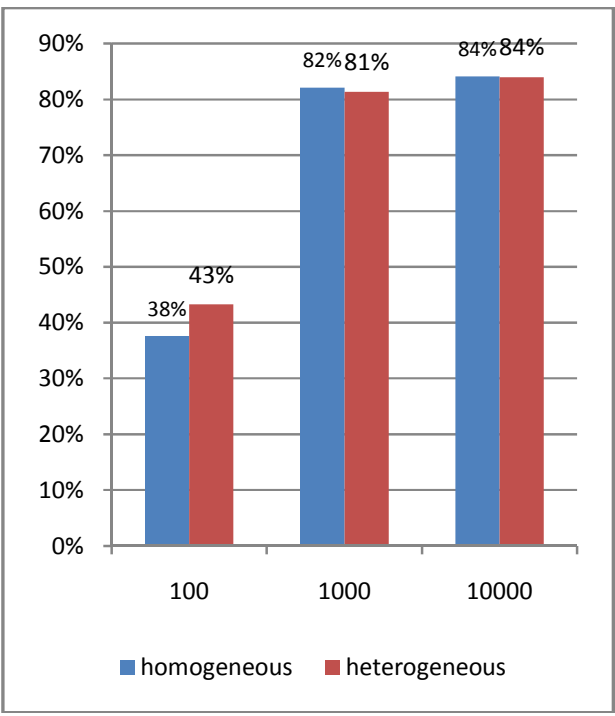

Figure 11.System speedup in case of 100 , 1000, and 10000 tasks running under 10 and 50 resources.

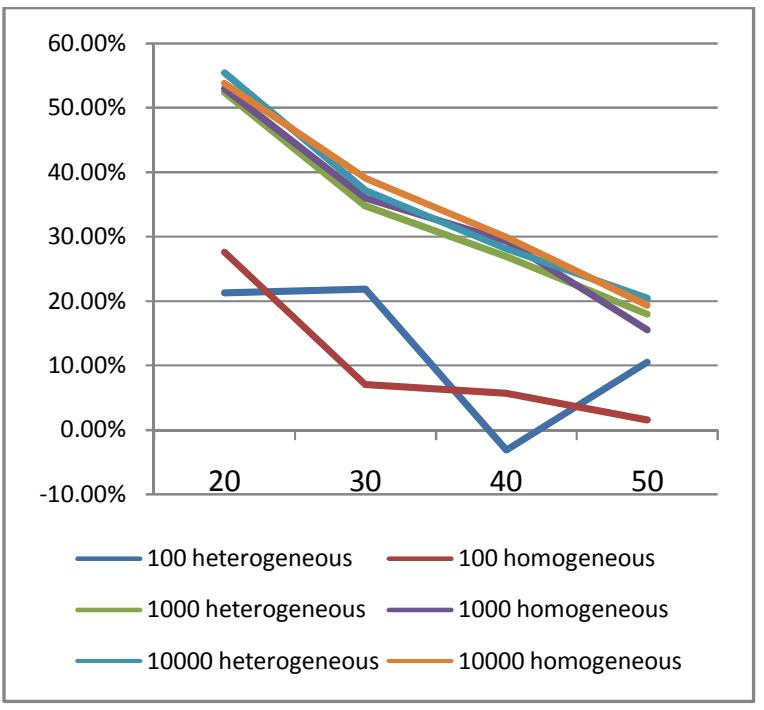

Figure 12.Average system speedup variation using 10, $20,30,40$, and 50 resources.

\subsubsection{Load balancing performance}

In order to study the load balancing performance of TunSys, which is the main goal, this section focuses on the percentage of executed tasks per resource. As expected, the system load is better distributed in case of homogeneous resources when considering the number of executed tasks per resource as shown in 0and 0 In addition, every time the number of resources is increased by 10 , the load balancing is improved especially when the system executes bigger number of tasks (0). This can lead to conclude that TunSys scales well.However how the system reacts in case of heterogeneous resourcesneeds to be verified. 
International Journal of Grid Computing \& Applications (IJGCA) Vol.8, No.1/2/3, September 2017

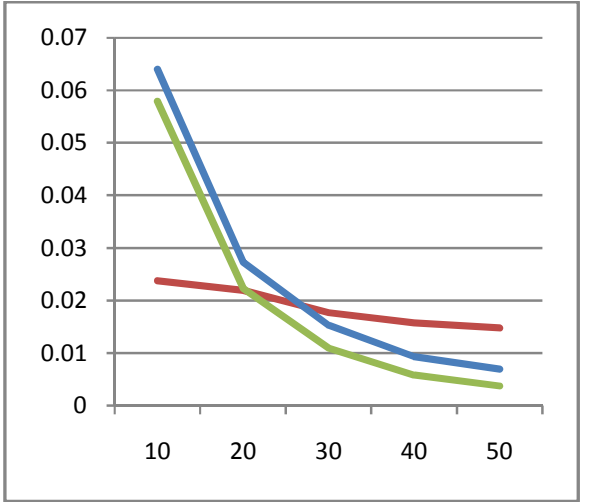

Figure 13. Mean square deviation of the percentage of executed tasks per resource in case of homogeneous resources.

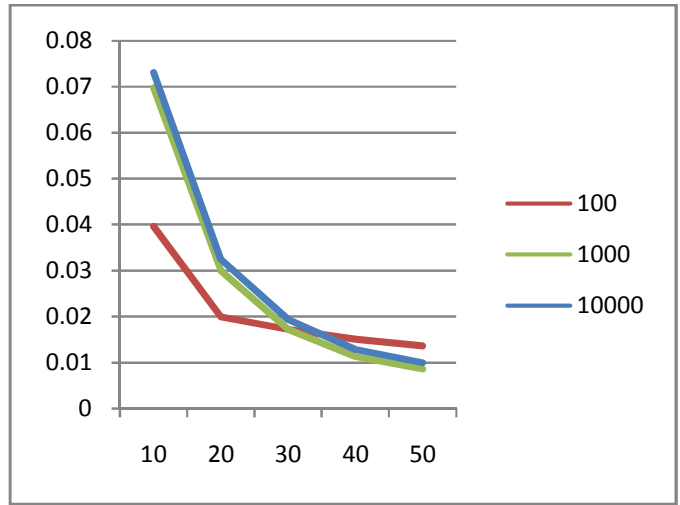

Figure 14 Mean square deviation of the percentage of executed tasks per resource in case of heterogeneous resources.

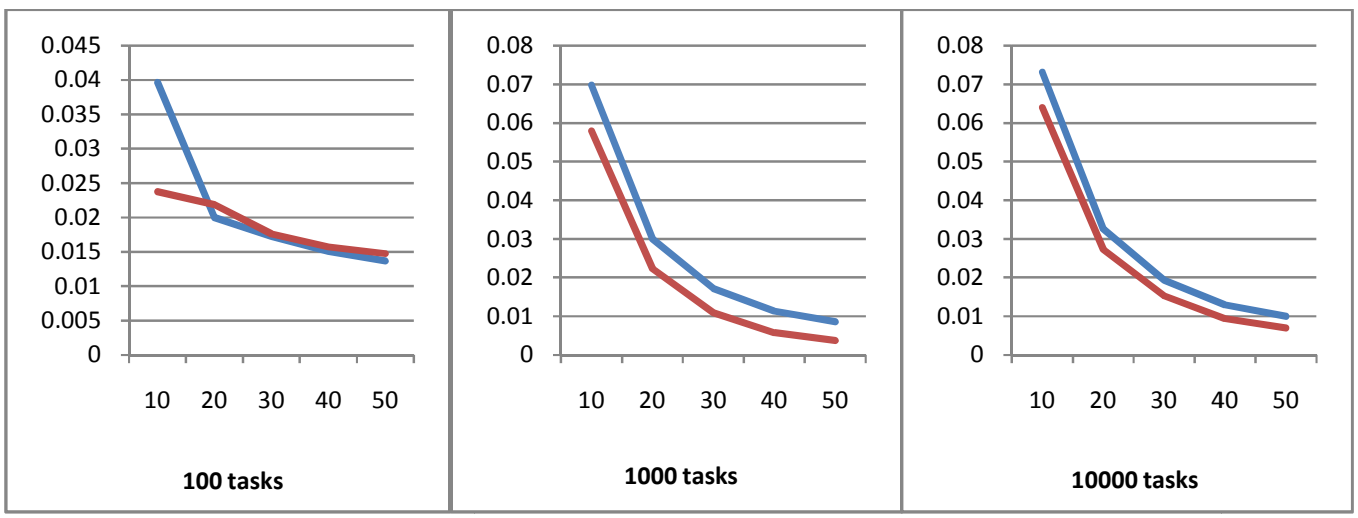

Figure 15. Mean square deviation of the percentage of executed tasks per resource in case of homogenous ( - ) vs. heterogeneous (_ ) resources with 100, 1000, and 10000 tasks.
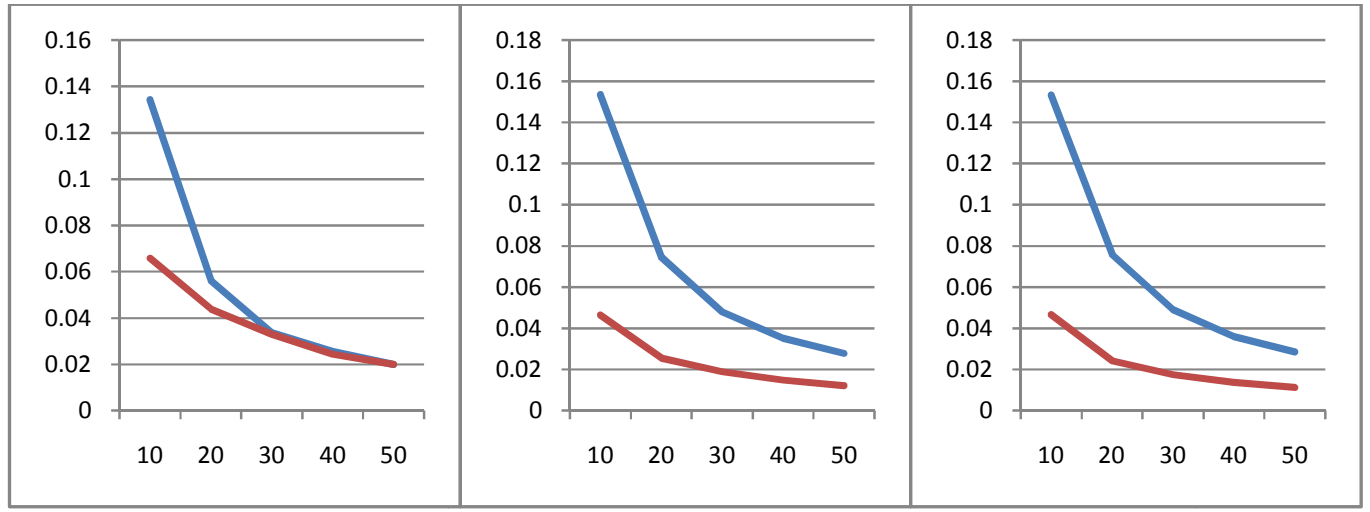

Figure .15.Average percentage of executed tasks per resourceconsidering resources with $\mathrm{PE}=2\left({ }_{-}\right)$vs. resources with $\mathrm{PE}=4$ ( $\longrightarrow$ ).

The 0 , presents the average percentage of executed tasks per resource. It compares the load distribution between most powerful resources $(\mathrm{PE}=4)$ and less powerful resources $(\mathrm{PE}=2)$. The results show that the load distribution is considering the resource performance represented by the 
International Journal of Grid Computing \& Applications (IJGCA) Vol.8, No.1/2/3, September 2017

number of processing elements (PE). The most powerful resources are executing more tasks (more than the double especially in case of 10 nodes) except in case of 100 tasks which is due to the limited number of tasks and the blind scheduling policy TunSysuses.

\section{CONCLUSION}

In this work, a topology-aware load balancing method for grid computing systems is proposed. Based on a polyhedron logical model with a sphere-like structure, the proposed method ensures a local neighborhood load balancing strategy using a combination of a local and a global thresholds. The proposed load balancing method is implemented and evaluated within a simple blind scheduling strategy, since the load balancing problem is the aim of this work. GridSim simulator isused for experimentalcomparison between TunSysand FCFS, DGA, and HGA through the makespan and mean square deviation metrics.

The comparative evaluation of TunSys with FCFS, DGA and HGA shows encouraging results.In fact, the system performance (measured through makespan) shows that TunSys outperforms FCFS, DGA, and in most cases HGA. In addition, it shows an excellent system scalability that can reach linear and supra-linear levels in case of 1000 and 10000 tasks. Furthermore, TunSys shows good load balancing performance in terms of system stability by taking advantage of resource heterogeneity, and the scalability of its load balancing approach.

Currently, the goal is to optimize the load balancing solution by integrating a better scheduling policy. In addition, TunSyswill be compared to other more recent works involving different logical models like star, tree, and peer-to-peer.

\section{REFERENCES}

[1] R. Buyya and S. Venugopal, "A Gentle Introduction to Grid Computing and Technologies," CSI Commun., vol. 2, no. July, pp. 1-19, 2005.

[2] Y. Huang, N. Bessis, P. Norrington, P. Kuonen, and B. Hirsbrunner, "Exploring decentralized dynamic scheduling for grids and clouds using the community-aware scheduling algorithm," Futur. Gener. Comput. Syst., vol. 29, no. 1, pp. 402-415, Jan. 2013.

[3] K. Yan, S. Wang, S. Wang, and C. Chang, "Towards a hybrid load balancing policy in grid computing system,” Expert Syst. Appl., vol. 36, no. 10, pp. 12054-12064, 2009.

[4] R. Buyya, S. Chapin, and D. DiNucci, "Architectural models for resource management in the grid," Grid Comput. 2000, pp. 18-35, 2000.

[5] J. Cao, D. P. Spooner, S. a. Jarvis, G. R. Nudd, and S. Augustin, "Grid load balancing using intelligent agents,” Futur. Gener. Comput. Syst., vol. 21, no. 1, pp. 135-149, Jan. 2005.

[6] Y. Li, Y. Yang, M. Ma, and L. Zhou, "A hybrid load balancing strategy of sequential tasks for grid computing environments," Futur. Gener. Comput. Syst., vol. 25, no. 8, pp. 819-828, Sep. 2009.

[7] A. Zomaya and Y. Teh, "Observations on using genetic algorithms for dynamic load-balancing," IEEE Trans. Parallel Distrib. Syst., vol. 12, no. 9, pp. 899-911, 2001.

[8] L. Bai, Y. L. Hu, S. Y. Lao, and W. M. Zhang, "Task scheduling with load balancing using multiple ant colonies optimization in grid computing," in Proceedings - 2010 6th International Conference on Natural Computation, ICNC 2010, 2010, vol. 5, pp. 2715-2719.

[9] F. Xhafa and A. Abraham, "Computational models and heuristic methods for Grid scheduling problems," Futur. Gener. Comput. Syst., vol. 26, no. 4, pp. 608-621, Apr. 2010.

[10] J. C. Patni, M. S. Aswal, A. Agarwal, and P. Rastogi, "A Dynamic and Optimal Approach of Load Balancing in Heterogeneous Grid Computing Environment," in Emerging ICT for Bridging the Future-Proceedings of the 49th Annual Convention of the Computer Society of India CSI, 2015, vol. 2, pp. 447-455.

[11] K. Q. Yan, S. C. Wang, C. P. Chang, and J. S. Lin, "A hybrid load balancing policy underlying grid computing environment," Comput. Stand. Interfaces, vol. 29, no. 2, pp. 161-173, Feb. 2007.

[12] N. J. Kansal and I. Chana, "Cloud Load Balancing Techniques : A Step Towards Green Computing,” IJCSI Int. J. Comput. Sci. Issues, vol. 9, no. 1, pp. 238-246, 2012. 
International Journal of Grid Computing \& Applications (IJGCA) Vol.8, No.1/2/3, September 2017

[13] G. Terzopoulos and H. Karatza, "Energy-efficient real-time heterogeneous cluster scheduling with node replacement due to failures," J. Supercomput., vol. 68, no. 2, pp. 867-889, Dec. 2013.

[14] H. Barkallah, M. Gzara, and H. Ben Abdallah, "A fully distributed Grid meta scheduling method for non dedicated resources," in International Conference on Parallel and Distributed Processing with Applications (ICPDPA'2014), IEEE WCCAIS'2014 Congress, 2014, no. 1, pp. 1-6.

[15] B. Yagoubi, "Load Balancing Strategy in Grid Environment," J. Inf. Technol. Appl., vol. 1, no. 4, pp. 285-296, 2007.

[16] M. S. B. Qureshi et al., "Survey on Grid Resource Allocation Mechanisms," J. Grid Comput., vol. 12, no. 2, pp. 399-441, Apr. 2014.

[17] Y. Zhu and L. M Ni, "A survey on grid scheduling systems, Technical Report SJTU_CS_TR_200309001,” 2013.

[18] J. Cao, "Self-organizing agents for grid load balancing," in Proceedings of the 5th IEEE/ACM International Workshop on Grid Computing, 2004, no. November, pp. 388-395.

[19] M. A. Salehi and H. Deldari, "Grid Load Balancing using an Echo System of Intelligent Ants.," in Parallel and Distributed Computing and Networks, 2006, pp. 47-52.

[20] M. A. Salehi, "Balancing Load in a Computational Grid Applying Adaptive, Intelligent Colonies of Ants," Informatica, vol. 33, no. 2, pp. 327-335, 2008.

[21] D. P. Spooner, S. a. Jarvis, J. Cao, S. Saini, and G. R. Nudd, "Local grid scheduling techniques using performance prediction,” IEE Proc. - Comput. Digit. Tech., vol. 150, no. 2, p. 87, 2003.

[22] R. Subrata, A. Y. Zomaya, and B. Landfeldt, "Artificial life techniques for load balancing in computational grids,” J. Comput. Syst. Sci., vol. 73, no. 8, pp. 1176-1190, 2007.

[23] L. He, S. A. Jarvis, D. Bacigalupo, D. P. Spooner, and G. R. Nudd, "Performance-Aware Load Balancing for Multiclusters," Parallel Distrib. Process. Appl. Second Int. ISPA 2004, vol. 3358, pp. 635-647, 2004.

[24] B. Yagoubi and Y. Slimani, "Dynamic Load Balancing Strategy for Grid Computing," Trans. Eng. Comput. Technol., vol. 13, pp. 260-265, 2006.

[25] K. Kandalla, H. Subramoni, A. Vishnu, and D. K. Panda, "Designing topology-aware collective communication algorithms for large scale InfiniBand clusters: Case studies with Scatter and Gather," 2010 IEEE Int. Symp. Parallel Distrib. Process. Work. Phd Forum, pp. 1-8, Apr. 2010.

[26] W.-C. Yeh and S.-C. Wei, "Economic-based resource allocation for reliable Grid-computing service based on Grid Bank,” Futur. Gener. Comput. Syst., vol. 28, no. 7, pp. 989-1002, Jul. 2012.

[27] M. Heidt, T. D, K. D, and B. Freisleben, "Omnivore: Integration of Grid Meta-Scheduling and Peer-to-Peer Technologies,” 2008 Eighth IEEE Int. Symp. Clust. Comput. Grid, pp. 316-323, May 2008.

[28] S. Rho, H. Chang, S. Kim, and Y. S. Lee, "An efficient peer-to-peer and distributed scheduling for cloud and grid computing," Peer-to-Peer Netw. Appl., Apr. 2014.

[29] R.-M. Chen and C.-M. Wang, "Project scheduling heuristics-based standard PSO for task-resource assignment in heterogeneous grid," in Abstract and Applied Analysis, 2011, vol. 2011.

[30] V. Lo, K. J. Windisch, W. Liu, and B. Nitzberg, "Noncontiguous processor allocation algorithms for mesh-connected multicomputers," Parallel Distrib. Syst. IEEE Trans., vol. 8, no. 7, pp. 712726, 1997.

[31] G. Levitin and Y.-S. Dai, "Optimal service task partition and distribution in grid system with star topology,” Reliab. Eng. Syst. Saf., vol. 93, no. 1, pp. 152-159, Jan. 2008.

[32] M.-C. Lee, F.-Y. Leu, and Y. Chen, "PFRF: An adaptive data replication algorithm based on startopology data grids," Futur. Gener. Comput. Syst., vol. 28, no. 7, pp. 1045-1057, Jul. 2012.

[33] C. Coti, T. Herault, and F. Cappello, "MPI applications on grids: A topology aware approach," Euro-Par 2009 Parallel Process. Lect. Notes Comput. Sci., vol. 5704, pp. 466-477, 2009.

[34] M. Swany and R. Wolski, "Building performance topologies for computational grids," Int. J. High Perform. Comput. Appl., vol. 18, no. 2, pp. 255-265, 2004.

[35] W. Gentzsch, "Grid Computing: A New Technology for the Advanced Web," in Advanced Environments, Tools, and Applications for Cluster Computing SE - 1, vol. 2326, D. Grigoras, A. Nicolau, B. Toursel, and B. Folliot, Eds. Springer Berlin Heidelberg, 2002, pp. 1-15.

[36] N. Fujimoto and K. Hagihara, "A comparison among grid scheduling algorithms for independent coarse-grained tasks,” 2004 Int. Symp. Appl. Internet Work. 2004 Work., pp. 674-680, 2004.

[37] P. E. GmbH, "Validations Manual 4.2," 2012. [Online]. Available: http://www.theseus-fe.com/.

[38] Z. Spakovszky, "MIT course: Introduction to Engineering Heat Transfer," 2012. .

[39] H. Barkallah, M. Gzara, and H. Ben Abdallah, "Dynamic and adaptive topology-aware load balancing for Grids," in FCST'2014, 2014. 
International Journal of Grid Computing \& Applications (IJGCA) Vol.8, No.1/2/3, September 2017

[40] R. Subrata, A. Y. Zomaya, B. Landfeldt, and S. Member, "Game-Theoretic Approach for Load Balancing in Computational Grids," IEEE Trans. Parallel Distrib. Syst., vol. 19, no. 1, pp. 66-76, 2008.

[41] M. A. Salehi, H. T. Yazdi, and M. R. A. Toutoonchi, "An optimal job selection method in load balancing algorithms of economical grids," in Innovations and Advanced Techniques in Systems, Computing Sciences and Software Engineering, 2008, pp. 362-365.

[42] R. Buyya and M. Murshed, "GridSim: a toolkit for the modeling and simulation of distributed resource management and scheduling for Grid computing," Concurr. Comput. Pract. Exp., vol. 14, no. 13-15, pp. 1175-1220, Nov. 2002.

[43] L. Kleinrock, Queueing Systems, Volume I: Theory. New York: Wiley Interscience, 1975.

\section{Authors}

B. Haitham received a master degree in Computer Science and multimedia from the University of Sfax, Tunisia. Currently, he is a Ph.D. student and a member of the Multimedia, Information systems and Advanced Computing Laboratory (Mir@cl), University of Sfax. His research interests include grid and cloud computing, scheduling and load balancing optimization.

M. Gzara received a $\mathrm{PhD}$ in automatic and industrial computing from the University of Lille 1, France. She is currently an Associate Professor in Computer Science at the Higher School of Computer Science and Mathematics of Monastir, University of Monastir, Tunisia. She is a member of the Multimedia, Information systems and Advanced Computing Laboratory (Mir@cl), University of Sfax. Her research interests include data mining techniques, Optimization, Parallelization, Distributed Compuattion and Information Retrieval.

H. Ben-Abdallah received a BS in Computer Science and BS in Mathematics from the University of Minnesota, MPLS, MN, a MSE and $\mathrm{PhD}$ in Computer and Information Science from the University of Pennsylvania, Philadelphia, PA. She worked at University of Sfax, Tunisia from 1997 until 2013. She is now full professor at the Faculty of Computing and Information Technology, King Abdulaziz University, Jeddah, Kingdom of Saudi Arabia. She is a member of the Multimedia,

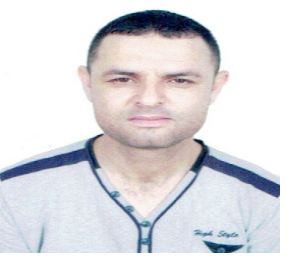
Information systems and Advanced Computing Laboratory (Mir@cl), University of Sfax. Her research interests include software design quality, reuse techniques in software and business process modeling. 